\title{
Groups Admitting a Kantor Family and a Factorized Normal Subgroup
}

\author{
DIRK HACHENBERGER \\ hachentuerger@math.ūni-aūgsbürg.ủe \\ Institut für Mathematik der Universität Augsburg, Universitätsstraße 14, D-86135 Augsburg
}

Communicated by: D. Jungnickel

Received May 12, 1995; Accepted October 26, 1995

Dedicated to Hanfried Lenz on the occasion of his 80th birthday

\begin{abstract}
We study the structure of a finite group $G$ admitting a Kantor family $\left(\mathcal{F}, \mathcal{F}^{*}\right)$ of type $(s, t)$ and a nontrivial normal subgroup $X$ which is factorized by $\mathcal{F} \cup \mathcal{F}^{*}$. The most interesting cases, giving necessary conditions on the structure of $G$ and the parameters $s$ and $t$, are those where a further Kantor family is induced in $X$, or where a partial congruence partition is induced in the factor group $G / X$. Most of the known finite generalized quadrangles can be constructed as coset geometries with respect to a Kantor family. We show that the parameters of a skew translation generalized quadrangle necessarily are powers of the same prime. Furthermore, the structure of nonabelian groups admitting a Kantor family consisting only of abelian members is considered.
\end{abstract}

Keywords: Generalized Quadrangle, Elation Generalized Quadrangle, Skew Translation Generalized Quadrangle, Kantor Family, 4-Gonal Family, Translation Net, Partial Congruence Partition, Group Coset Geometry.

\section{Introduction}

Most of the known families of finite generalized quadrangles are elation generalized quadrangles, i.e., they admit a group $G$ of collineations fixing a base point $\pi$ and all lines incident with $\pi$, and acting sharply transitively on the set of points which are not joined with $\pi$. (The standard reference for generalized quadrangles is the monograph [14] of S. E. Payne and J. A. Thas. For the theory of finite groups we refer to B. Huppert [7]).

In [10], W. M. Kantor has shown that the existence of elation generalized quadrangles is equivalent to the existence of a certain set of subgroups (a 4-gonal family or a Kantor family) of the elation group $G$, and that all these quadrangles can be represented as coset geometries with respect to such families.

Using this general construction scheme, many new series of finite generalized quadrangles have been constructed during the 1980s (the reader is refered to Payne [13] for a survey). All these new examples belong to the particular class of skew translation generalized quadrangles, i.e., in each case the corresponding elation group $G$ admits a full group of symmetries about $\pi$ (a subgroup fixing every point joined with $\pi$ and having maximal cardinality with respect to this property). The existence of such a symmetry group is reffected in the Kantor family.

On the other hand, necessary conditions on the structure of groups admitting a Kantor family are studied in some papers: It is conjectured in Kantor [11] that the elation group 
$G$ necessarily is a group of prime power order (which implies that the parameters $s$ and $t$ of the corresponding quadrangle are powers of the same prime dividing the cardinality $|G|$ of $G$ ). In his fundamental paper [3], D. Frohlardi among other things proved the validity of Kantor's conjecture provided $t \geq s$ (observe that the rôle of $s$ and $t$ is interchanged in [3]). In Chen and Frohardt [1] and Hachenberger [5], the structure of $G$ is studied under the additional assumption that some members of a Kantor family in $G$ are normal subgroups of $G$.

In the present paper the work towards the latter direction is continued, i.e., we study the structure of a group $G$ admitting a Kantor family $\left(\mathcal{F}, \mathcal{F}^{*}\right)$ of type $(s, t)$ and a nontrivial normal subgroup $X$ which is factorized by $\mathcal{F} \cup \mathcal{F}^{*}$ (see Section 2). The most interesting cases, including Kantor families which coordinatize a skew translation generalized quadrangle, are those where a further Kantor family is induced in the subgroup $X$, or where a partial congruence partition is induced in the factor group $G / X$. Therefore, due to an additional assumption on $G$ and $\left(\mathcal{F}, \mathcal{F}^{*}\right)$, similar to [5], we meet situations where further geometries occur (translation nets are obtained as coset geometries from partial congruence partitions).

Our paper is set up as follows: In Section 2 general results are presented. Although we did not succeed in proving that $G$ necessarily has prime power order provided it admits a nontrivial normal subgroup which is factorized by $\mathcal{F} \cup \mathcal{F}^{*}$, we have found interesting results showing that the parameters and the structure of the elation group are very restricted, e.g., see Corollary 2.6, the parameters of a skew translation generalized quadrangle necessarily are both powers of the same prime.

In Section 3, we study nonabelian groups $G$ admitting a Kantor family consisting only of abelian members. In this case the center of $G$ is factorized. As far as the author is aware of, this indeed holds in all known examples of skew translation generalized quadrangles.

\section{General Results}

Let $s, t>1$ be integers and let $G$ be a (multiplicatively written) group of order $s^{2} t$. A Kantor family or a 4-gonal family of type $(s, t)$ in $G$ is a pair $\left(\mathcal{F}, \mathcal{F}^{*}\right)$ where $\mathcal{F}$ and $\mathcal{F}^{*}$ are sets of subgroups of $G$ satisfying the following axioms (if $X$ is a set then $|X|$ denotes the cardinality of $X)$ :

$(\mathrm{K} 1)|\mathcal{F}|=\left|\mathcal{F}^{*}\right|=t+1$

(K2) for all $A \in \mathcal{F}$ there exists exactly one $A^{*} \in \mathcal{F}^{*}$ such that $A \subseteq A^{*}$;

(K3) $|A|=s$ and $\left|A^{*}\right|=s t$ for all $A \in \mathcal{F}$;

(K4) $A \cap B^{*}=\{1\}$ for any two different members $A$ and $B$ of $\mathcal{F}$;

(K5) $A B \cap C=\{1\}$ for any pairwise different members $A, B, C$ of $\mathcal{F}$.

Throughout, we assume that $G$ admits a $\operatorname{Kantor}$ family $\left(\mathcal{F}, \mathcal{F}^{*}\right)$ of type $(s, t)$. Let $\mathcal{S}:=$ $\mathcal{F} \cup \mathcal{F}^{*}$. We consider the structure of $G$ under the additional assumption that there exists a nontrivial subgroup $X$ of $G$ satisfying the following property:

(F) $(U \cap X)(V \cap X)=X$ for all $U, V \in \mathcal{S}$ satisfying $U V=G$.

A nontrivial subgroup $X$ satisfying $(\mathrm{F})$ is called an $\mathcal{S}$-factor of $G$. We say that $X$ is factorized by $\mathcal{S}$. 
Our approach is motivated by a situation in Hachenberger [5], where Kantor families with some member $A$ of $\mathcal{F}$ being a normal subgroup of $G$ are studied, and by an approach studying partial congruence partitions in Hachenberger [4] (a definition is given subsequent to Proposition 2.2 of the present paper). The first result is a straightforward generalization of a part of Proposition 2.2 in [5].

LEMMA 2.1 Let $X$ be an $\mathcal{S}$-factor of $G$. Then there exist integers $\sigma \geq 1$ and $\tau \geq 1$ such that the cardinality $|X|$ of $X$ is equal to $\sigma^{2} \tau$, and $|A \cap X|=\sigma$ and $\left|A^{*} \cap X\right|=\sigma \tau$ for all $A \in \mathcal{F}$.

Proof. First, observe that the axioms imply $A B^{*}=G=A^{*} B^{*}$ and $A B \neq G$ for any two different members $A$ and $B$ of $\mathcal{F}$. Now, let $A, B$, and $C$ be any pairwise different members of $\mathcal{F}$. Then, by (K4), (F), and the well known property that $|U V|=|U| \cdot|V| /|U \cap V|$ for any two subgroups $U$ and $V$ of some finite group, we have

$$
|X|=|A \cap X| \cdot\left|B^{*} \cap X\right|=|A \cap X| \cdot\left|C^{*} \cap X\right|=|B \cap X| \cdot\left|C^{*} \cap X\right| .
$$

Thus the intersection numbers $|A \cap X|$ respectively $\left|A^{*} \cap X\right|$ are constant for all $A \in \mathcal{F}$, say $\sigma$ and $\sigma \tau$, respectively (observe that $A \cap X$ is a subgroup of $A^{*} \cap X$ ). Therefore, the cardinality of $X$ is equal to $\sigma^{2} \tau$.

From now on we assume that $X$ is an $\mathcal{S}$-factor of $G$. Let $\mathcal{F}_{X}:=\{U \cap X \mid U \in \mathcal{F}\}$, $\mathcal{F}_{X}^{*}:=\left\{U \cap X \mid U \in \mathcal{F}^{*}\right\}$, and $\mathcal{S}_{X}:=\mathcal{F}_{X} \cup \mathcal{F}_{X}^{*}$. Furthermore, with $\sigma$ and $\tau$ as in Lemma 2.1, let $(\sigma, \tau)$ be the type of $X$.

Proposition 2.2 Let $X$ be an $\mathcal{S}$-factor of type $(\sigma, \tau)$ in $G$. Then necessarily one of the following cases occurs:

(2.2.1) $\sigma=1,|X|=\tau \leq t$, and $X$ is a subgroup of $\bigcap_{A \in \mathcal{F}} A^{*}$.

(2.2.2) $\sigma>1, \tau=t$, and $\left(\mathcal{F}_{X}, \mathcal{F}_{X}^{*}\right)$ is a Kantor family in $X$ of type $(\sigma, t)$.

Proof. If $\sigma=1$, the first part follows immediately from Lemma 2.1.

Assume therefore that $\sigma>1$. In order to prove (2.2.2), we first observe that $\tau>1$ : Otherwise, we have $A \cap X=A^{*} \cap X$ for all $A \in \mathcal{F}$, whence $X=(A \cap X)(B \cap X)$ contains $C \cap X$ for any three different members $A, B, C$ of $\mathcal{F}$. Since $X$ is a nontrivial subgroup of $G$, this is a contradiction to (K5).

Consequently, the pair $\left(\mathcal{F}_{X}, \mathcal{F}_{X}^{*}\right)$ consists of subgroups of $X$ satisfying the axioms (K2) to (K5) with respect to the parameter pair $(\sigma, \tau)$. Moreover, $\left|\mathcal{F}_{X}\right|=\left|\mathcal{F}_{X}^{*}\right|=t+1$. It therefore remains to show that $\tau=t$. Of course, $\tau \leq t$, since for different $A, B \in \mathcal{F}$, the conditions imply that $\left|A^{*} \cap B^{*} \cap X\right|=\frac{(\sigma \tau)^{2}}{\sigma^{2} \tau}=\tau$, and $\left|A^{*} \cap B^{*}\right|=\frac{(s t)^{2}}{s^{2} t}=t$. On the other hand, Lemma 2 of Frohardt [3] gives $t \leq \tau$. We therefore have $\tau=t$, and everything is proved.

If $\sigma>1$, then the induced Kantor family in $X$ gives rise to a subquadrangle (with parameters $\sigma$ and $t$ ) of the original generalized quadrangle coordinatized by $G$ and $\left(\mathcal{F}, \mathcal{F}^{*}\right)$. Moreover, this subquadrangle is an elation generalized quadrangle with respect to $X$ and $\left(\mathcal{F}_{X}, \mathcal{F}_{X}^{*}\right)$. 
We furthermore remark that the case where $X$ is a normal subgroup of $G$ and has type $(1, t)$ is of particular interest, because then the generalized quadrangle via Kantor's construction corresponding to $\left(\mathcal{F}, \mathcal{F}^{*}\right)$ is a skew translation generalized quadrangle (STGQ) with $X=$ $\bigcap_{A \in \mathcal{F}} A^{*}$ being a full group of symmetries about the base point (see [14], p. 166).

We next assume that $X$ is a normal subgroup of $G$ and consider the factor group $G / X$. Let $\mathcal{S}^{X}:=\{U X / X \mid U \in \mathcal{S}\}$. The following result again is similar to the situation met in Proposition 2.2 of [5]. We need a further definition: Let $H$ be a finite group of order $h^{2}>1$. A partial congruence partition (PCP) of type $(h, r)$ in $H$ is a set $\mathcal{H}$ of $r \geq 3$ subgroups of $H$ satisfying:

(P1) $|U|=h$ for all $U \in \mathcal{H}$;

(P2) $U \cap V=\{1\}$ for any two different members $U$ and $V$ of $\mathcal{H}$.

The parameter $r$ is called the order of $\mathcal{H}$. After the fundamental paper [8] of D. Jungnickel, the existence of partial congruence partitions (and generalizations) has been intensively studied in the past few years, because, similar to Kantor families, they are equivalent to a certain class of finite geometries, namely translation nets. The reader is refered to the recent survey Hachenberger and Jungnickel [6].

Proposition 2.3 Let $X$ be a normal $\mathcal{S}$-factor of $G$ of type $(\sigma, \tau)$, and assume that $\tau=t$. Then $\mathcal{S}^{X}=\{A X / X \mid A \in \mathcal{F}\}$ is a partial congruence partition of type $\left(\frac{s}{\sigma}, t+1\right)$ in $G / X$.

Proof. First, it follows immediately that $G / X$ has cardinality $\frac{s^{2}}{\sigma^{2}}$, and that $A X / X$ and $A^{*} X / X$ are equal with cardinality $\frac{s}{\sigma}$. Next, let $A$ and $B$ be different members of $\mathcal{F}$. If $a X=b X$ for $a \in A$ and $b \in B$, then $a^{-1} b \in X=(A \cap X)\left(B^{*} \cap X\right)$ (here we have used that $X$ is an $\mathcal{S}$-factor). Now there exist elements $a^{\prime}$ and $b^{\prime}$ in $A \cap X$ and $B^{*} \cap X$, respectively, such that $a^{-1} b=a^{\prime} b^{\prime}$. From (K4) it follows that $a^{-1}=a^{\prime}$ and $b=b^{\prime}$ whence $a X=X=b X$. This shows that $A X / X \cap B X / X=X / X$. As $X$ is not equal to $G$, we have $\sigma<s$ and therefore $\mathcal{S}^{X}$ has cardinality $|\mathcal{F}|=t+1$, whence everything is proved.

Applying inequalities on the order of a partial congruence partition and on the parameters of a finite generalized quadrangle, the situation in Proposition 2.3 can be further specified.

PROPOSITION 2.4 Let $X$ be a normal $\mathcal{S}$-factor of $G$ of type $(\sigma, \tau)$, and assume that $\tau=t$. Then the following holds:

(2.4.1) $t \leq \frac{s}{\sigma}$,

(2.4.2) $\sigma^{2} \leq s$,

(2.4.3) $\sigma \leq t$.

Moreover, equality holds in (2.4.1) if, and only if $\mathcal{S}^{X}$ is a partition of $G / X$ in the group theoretical sense (i.e., any nonidentity element in $G / X$ lies in exactly one member of $\mathcal{S}^{X}$ ). In this case $G / X$ necessarily is elementary abelian, and, $t$ and $s$ are powers of the same prime. 
Furthermore, equality in (2.4.2) or (2.4.3) implies equality in (2.4.1), (2.4.2), and (2.4.3). In this case $\left(\mathcal{F}, \mathcal{F}^{*}\right)$ and $\left(\mathcal{F}_{X}, \mathcal{F}_{X}^{*}\right)$ are of type $\left(t^{2}, t\right)$ and $(t, t)$ respectively, $t$ is a prime power, and $\mathcal{S}^{X}$ is a partition of $G / X$.

Proof. The validity of (2.4.1) is a well-known fact from the theory of translation nets (see e.g., [6]). In order to prove the other inequalities, the fundamental inequality of Higman, stating that the parameters $a$ and $b$ of a finite generalized quadrangle satisfy $a \leq b^{2}$ and $b \leq a^{2}$ provided $a, b \geq 2$ (see [14]) is needed. In combination with (2.4.1) it gives $s \leq t^{2} \leq \frac{s^{2}}{\sigma^{2}}$, from which (2.4.2) follows. Moreover, if $t<\sigma$, then (2.4.2) implies $t^{2}<\sigma^{2} \leq s$, a contradiction, whence (2.4.3) holds.

Now, if equality holds in (2.4.2), then (2.4.1) implies $t \leq \sigma$, whence equality occurs in (2.4.3) and in (2.4.1). Finally, if $\sigma=t$, i.e., equality holds in (2.4.3), then Higman's inequality implies equality in (2.4.2), hence in (2.4.1).

We mention that these inequalities already hold for arbitrary finite generalized quadrangles with parameters $s$ and $t$ admitting a proper subquadrangle (see 2.2.2 in [14]).

We next prove the statements of the proposition concerning the elation group $G$ and the normal $\mathcal{S}$-factor $X$ : If equality holds in $(2.4 .1)$ then, due to the fact that $\mathcal{S}^{X}$ is a partial congruence partition in $G / X$, we have

$$
\sum_{U \in \mathcal{S}^{X}}(|U|-1)+1=\left(\frac{s}{\sigma}+1\right)\left(\frac{s}{\sigma}-1\right)+1=|G / X| .
$$

Thus $\mathcal{S}^{X}$ is a partition in $G / X$. It is well known that $\mathcal{S}^{X}$ coordinatizes a translation plane and that $G / X$ necessarily is elementary abelian. Now, if $\sigma=1$ then $s=t$, whence Theorem 1 of Frohardt [3] implies that $s$ and $t$ are powers of the same prime. If $\sigma>1$, the same result applied to the subgroup $X$ with parameters $t \geq \sigma$ gives that $\sigma$ and $t$ are powers of the same prime. Since $\frac{s}{\sigma}=t$ by assumption, we obtain that $s$ and $t$ are powers of the same prime. The rest is clear.

Using further results of Frohardt [3], we are now able to prove the following theorem.

THEOREM 2.5 Let $G$ be a group of order $s^{2} t$ admitting a Kantor family $\left(\mathcal{F}, \mathcal{F}^{*}\right)$ of type $(s, t)$ and a normal $\mathcal{S}$-factor $X$ of type $(\sigma, \tau)$ with $\tau=t$ (where $\left.\mathcal{S}=\mathcal{F} \cup \mathcal{F}^{*}\right)$. Then necessarily one of the following cases occurs:

(2.5.1) $G$ is a group of prime power order (say for the prime $p$ ).

(2.5.2) $\sigma>1,|G|$ has exactly two prime divisors (say $p$ and $q$ ), and $X$ is a Sylow subgroup of $G$ (say for the prime $q$ ).

Proof. We first show that $\frac{s}{\sigma}$ is a prime power: It is known that $\mathcal{S}^{X}$ induces a PCP of type $(\sqrt{|\bar{P}|}, t+1)$ in any Sylow subgroup $P$ of $G / X$. (This was first proved by D. Frohardt in [2] and is similar to the proof of Lemma 4 in Frohardt [3]; for generalizations of PCPs it is proved in Jungnickel [9].) Therefore, if $\frac{s}{\sigma}$ has at least two prime divisors, we may choose $P$ such that $|P|<\sqrt{|G / X|}=\frac{s}{\sigma}$. But then (see e.g. Theorem 2.2 in [6]) $t \leq \sqrt{|P|}<\sqrt{\frac{s}{\sigma}}$, whence $t^{2}<\frac{s}{\sigma} \leq s$. This is a contradiction to Higman's inequality. Thus $\frac{s}{\sigma}$ is a prime power. 
From now on, let $p$ be the unique prime divisor of $\frac{s}{\sigma}$. By Lemma 5 and Lemma 6 of Frohardt [3], the parameters $a$ and $b$ of a Kantor family are powers of the same prime, provided $b \geq a$ or $a$ has exactly one prime divisor. Thus, if $\sigma=1$, the first part of the proof implies that $s$ is a prime power, whence we have case (2.5.1). Assume next that $\sigma>1$. By Proposition $2.2,\left(\mathcal{F}_{X}, \mathcal{F}_{X}^{*}\right)$ is a Kantor family of type $(\sigma, t)$ in $X$, and by (2.4.3) we have that $t \geq \sigma$. Thus Frohardt's result implies that $t$ and $\sigma$ are powers of the same prime (say $q$ ), and $X$ is a $q$-group.

Altogether, we have that $|G|$ has only the prime divisors $p$ and $q$. In the case where $p \neq q$, we furthermore have that $X$ is a $q$-Sylow subgroup of $G$, which by the normality of $X$ is unique. Thus, everything is proved.

There is no example known with case (2.5.2) holding. Unfortunately, we were not able to exclude this case, yet. However, case (2.5.1) actually occurs in all examples of STGQ with $X$ being a full group of symmetries about the base point. Indeed, Theorem 2.5 answers a question posed in Payne [12] on such quadrangles:

\section{COROLLARY 2.6 The parameters of an STGQ are powers of the same prime.}

Proof. By definition, the elation group $G$ of an STGQ with parameters $s$ and $t$ admits a full group of symmetries $X$, which is equal to $\bigcap_{A \in \mathcal{F}} A^{*}$ and has cardinality $t$ (where $\left(\mathcal{F}, \mathcal{F}^{*}\right)$ is the Kantor family coordinatizing the quadrangle). Thus, $X$ is factorized by $\mathcal{F} \cup \mathcal{F}^{*}$ and has type $(1, t)$. We therefore have case (2.5.1) of Theorem 2.5 .

We remark that in the case where $\sigma=1$ and $\tau=t$ it is further known (see [12] and [14]) that necessarily $t \leq s$, and, if $t<s$, then there exist integers $n, a \geq 1$ such that $s=p^{n(a+1)}$ and $t=p^{n a}$. (However, the only known examples have $a=1$.)

We conclude this section with a further application of Theorem 2.5, stating that the parameters of a Kantor family are very restricted provided there exist nontrivial normal $\mathcal{S}$-factors $X$ and $Y$ such that $X$ is a proper subgroup of $Y$.

THEOREM 2.7 Let $G$ be a group of order $s^{2} t$ admitting a Kantor family $\left(\mathcal{F}, \mathcal{F}^{*}\right)$ of type $(s, t)$ and two normal $\mathcal{S}$-factors $X$ and $Y$ of types $\left(\sigma_{X}, t\right)$ and $\left(\sigma_{Y}, t\right)$, where $\mathcal{S}=\mathcal{F} \cup \mathcal{F}^{*}$. Assume that $X$ is a proper subgroup of $Y$. Then necessarily $\sigma_{X}=1, \sigma_{Y}=t, s=t^{2}$, and $|G|=t^{5}$ is a prime power. Moreover, the generalized quadrangle corresponding to $\left(\mathcal{F}, \mathcal{F}^{*}\right)$ is an $S T G Q$ of type $\left(t^{2}, t\right)$ with $X$ as full group of symmetries about the base point.

Proof. Theorem 2.5 implies that $G$ has prime power order, since in case (2.5.2) the $\mathcal{S}$-factor is unique.

An application of (2.4.1) and (2.4.2) to $(G, Y)$ gives $t \leq \frac{s}{\sigma_{Y}}$ and $\sigma_{Y} \leq t$; applying (2.4.1) to $(Y, X)$ gives $t \leq \frac{\sigma_{Y}}{\sigma_{X}}$ (observe that $\sigma_{Y}>\sigma_{X}$ by assumption, and that $\left(\mathcal{F}_{Y}, \mathcal{F}_{Y}^{*}\right)$ is a Kantor family of type $\left(\sigma_{Y}, t\right)$ in $\left.Y\right)$. Altogether we obtain that $\sigma_{X}=1$ and that $\sigma_{Y}=t$. As equality holds in (2.4.3) for $(G, Y)$, we have that $\left(\mathcal{F}, \mathcal{F}^{*}\right)$ has type $\left(t^{2}, t\right)$. The rest is clear. 
We finally remark that in the situation of Theorem 2.7, necessarily, $G / Y$ and $Y / X$ are elementary abelian of order $t^{2}$. Furthermore, there does not exist a proper sequence $X \subset$ $Y \subset Z$ of normal $\mathcal{S}$-factors in $G$, where the parameter $\tau_{X}$ is equal to $t$. Moreover, similar to the proof of Proposition 2.4, the pure combinatorial part of Theorem 2.7 follows from 2.2 .2 in [14].

\section{Abelian Kantor Families}

In this section we consider the structure of groups admitting an abelian Kantor family, i.e., a Kantor family consisting only of abelian groups. This assumption likewise is motivated by Proposition 2.2 in [5] and Section 3 in [4]. Throughout we use the same notation as in Section 2.

Since abelian groups admitting a Kantor family are necessarily elementary abelian (see e.g. [1]), we restrict our attention to nonabelian groups.

PROPOSITION 3.1 Let $G$ be a nonabelian group admitting an abelian Kantor family of type $(s, t)$. Let $X$ be the center of $G$. Then $X$ is a normal $\mathcal{S}$-factor of type $(\sigma, \tau)$ with $\tau=t$. Moreover, $G$ necessarily is a group of prime power order.

Proof. The first part is already proved in the course of Proposition 2.2 in [5] (here $t=\tau$ holds since $A^{*} \cap B^{*}$ centralizes $A^{*} B^{*}=G$, whence it is a subgroup of $X$ ).

In order to prove that $|G|$ is a prime power, it is sufficient to show that case (2.5.2) in Theorem 2.5 cannot occur. But this is clear, because otherwise (with the notation as in Theorem 2.5) $X$ is the unique $q$-Sylow subgroup, and, as $X$ is the center of $G$ and a complement of any $p$-Sylow subgroup $P$ of $G$, we see that $G$ is the direct product of $P$ with $X$. But then $X$ is isomorphic to the direct product of the center of $P$ with $X$. This gives a contradiction since the center of a finite $p$-group is not equal to $\{1\}$.

Next, we consider the partial congruence partition $\mathcal{S}^{X}$ in the factor group $G / X$. Then clearly every member of $\mathcal{S}^{X}$ is abelian. Thus, the Factorization Lemma in Hachenberger [4] can be applied to the center $Z$ of $G / X$, i.e., we have

$\left(\mathrm{F}^{\prime}\right) Z=(U \cap Z)(V \cap Z)$ for all different members $U$ and $V$ of $\mathcal{S}^{X}$.

In particular, if $G / X$ is nonabelian, then $Z$ is a proper subgroup of $G / X$. In this case it is easy to see that the subgroup $Y$ of $G$ containing $X$ and corresponding to $Z$ is a normal $\mathcal{S}$-factor of $G$, whence we have the situation of Theorem 2.7 with $X$ and $Y$ building the upper central series of $G$ (see also the remark subsequent to Theorem 2.7).

(Indeed, one can more generally show that the subgroups $W$ of $G / X$ satisfying $\left(\mathrm{F}^{\prime}\right)$ correspond bijectively to the $\mathcal{S}$-factors of $G$ containing $X$. Furthermore, if $X$ is an $\mathcal{S}$ factor of type $(\sigma, t)$, by Theorem 2.7 a necessary condition for the existence of a nontrivial subgroup $W$ of $G / X$ satisfying $\left(\mathrm{F}^{\prime}\right)$ is that $\sigma=1$.)

Next, we consider the case where $G / X$ is abelian, but not elementary abelian. Then the 
Factorization Lemma of [4] can be applied to the largest elementary abelian subgroup $\Omega:=\left\{g X \in G / X \mid(g X)^{p}=X\right\}$ of $G / X$ (where $p$ denotes the unique prime divisor of $|G|)$. Thus Theorem 2.7 holds in $G$ with $X$ bcing the center of $G$ and with $Y$ being the subgroup of $G$ corresponding to $\Omega$.

Using again a result of Frohardt [3], one can say more with respect to the latter situation: Observing that $X$ is of type $(1, t)$, we have that $A^{*} \cap B^{*}$ is equal to the center $X$ of $G$ for any two different members $A, B$ of $\mathcal{F}$. By assumption, $G / X$ is abelian. Furthermore, the validity of $\left(\mathrm{F}^{\prime}\right)$ shows that $\Omega$ does not contain $A X / X$ for any $A \in \mathcal{F}$, whence, due to the definition of $\Omega$, the exponent of $A X / X$ is at least $p^{2}$. In particular, $A X / X$ and $A$ are not elementary abelian for all $A \in \mathcal{F}$. Consequently, we have case (b) of Theorem 2 in [3] which states that necessarily $X$ is elementary abelian, that $G$ is a 2 -group (!), and that all elements of $\mathcal{F}$ are isomorphic of exponent 4.

Summarizing, we have proved the following theorem.

THEOREM 3.2 Let $G$ be a nonabelian group admitting an abelian Kantor family $\left(\mathcal{F}, \mathcal{F}^{*}\right)$ of type $(s, t)$. Let $\mathcal{S}:=\mathcal{F} \cup \mathcal{F}^{*}$ and let $X$ be the center of $G$. Then $X$ is an $\mathcal{S}$-factor, $G$ necessarily is a group of prime power order (say for the prime $p$ ), and necessarily one of the following cases occurs:

(3.2.1) $G / X$ is elementary abelian.

(3.2.2) $G$ has class 3, and the members of the upper central series of $G$ are normal $\mathcal{S}$ factors. Moreover, $\left(\mathcal{F}, \mathcal{F}^{*}\right)$ is of type $\left(t^{2}, t\right)$, and the generalized quadrangle corresponding to $G$ and $\left(\mathcal{F}, \mathcal{F}^{*}\right)$ is an $S T G Q$ with $X$ being a full group of symmetries about the base point.

(3.2.3) $G$ is a 2-group of class $2, G / X$ is abelian but not elementary abelian, and the subgroup $Y:=\left\{g \in G \mid(g X)^{2}=X\right\}$ is a normal $\mathcal{S}$-factor properly containing $X$. Moreover, $\left(\mathcal{F}, \mathcal{F}^{*}\right)$ is of type $\left(t^{2}, t\right)$, and the generalized quadrangle corresponding to $G$ and $\left(\mathcal{F}, \mathcal{F}^{*}\right)$ is an $S T G Q$ with $X$ being a full group of symmetries about the base point.

We finally remark that we do not know whether (3.2.2) and (3.2.3) actually can occur. However, in all known examples of STGQs (3.2.1) holds, with type equal to $(t, t)$ or $\left(t^{2}, t\right)$.

\section{Acknowledgements}

The author thanks the referees for their valuable suggestions which improved some details of the paper.

\section{References}

1. X. Chen and D. Frohardt, Normality in a Kantor family, Joumal of Combinatorial Theory A, Vol. 64 (1993) pp. 130-136.

2. D. Frohardt, Groups with a large number of large disjoint subgroups, Journal of Algebra, Vol. 107 (1987) pp. 153-159.

3. D. Frohardt, Groups which produce generalized quadrangles, Journal of Combinatorial Theory A, Vol. 48 (1988) pp. 139-145.

4. D. Hachenberger, On the existence of translation nets, Journal of Algebra, Vol. 152 (1992) pp. $207-229$. 
5. D. Hachenberger, On finite elation generalized quadrangles with symmetries, (1994) Journal of the London Mathematical Society, Vol. 53 (1996), 397-406.

6. D. Hachenberger and D. Jungnickel, Translation nets: A survey, Discrete Mathematics, Vol. 106/107 (1992) pp. 231-242.

7. B. Huppert, Endliche Gruppen I, Springer, Berlin (1967).

8. D. Jungnickel, Existence results for translation nets, in: Finite Geometries and Designs, (Cameron, Hirschfeld and Hughes, eds.) LMS Lecture Notes, Cambridge Univ. Press, Cambridge, 49 (1981) pp. 172196.

9. D. Jungnickel, Existence results for translation nets, II, Journal of Algebra, Vol. 122 (1989) pp. 288-298.

10. W. M. Kantor, Generalized quadrangles associated with $G_{2}(q)$, Journal of Combinatorial Theory A, Vol. 29 (1980) pp. 212-219.

11. W. M. Kantor, Generalized polygons, SCABs and GABs, In: Buildings and the Geometry of Diagrams, (L. A. Rosati, ed.), LNMS 1181 , Springer, Berlin (1984) pp. 79-158.

12. S. E. Payne, An essay on skew translation generalized quadrangles, Geometriae Dedicata, Vol. 32 (1989) pp. 93-118.

13. S. E. Payne, A census of finite generalized quadrangles, In: Finite Geometries, Buildings and related Topics, (Kantor, Liebler, Payne, Shult, eds.), Clarendon Press, Oxford (1990) pp. 29-36.

14. S. E. Payne and J. A. Thas, Finite Generalized Quadrangies, Pitman, Research Notes in Mathematics 110 , Boston (1984). 